\title{
METHODS OF COMPLEX OBJECT'S TRANSFER FUNCTION CALCULATION FOR DISTRIBUTED CONTROL SYSTEM
}

\author{
Alexander V. Martirosyan, Karina V. Martirosyan and Eduard G. Yanukyan \\ North-Caucasian Federal University, Branch in Pyatigorsk, \\ Russian Federation, 357500, Pyatigorsk, 40 let Oktyabrya ave., 56A, Russia
}

Received 2014-08-07; Revised 2014-09-08; Accepted 2014-09-16

\begin{abstract}
One of the ways of solving the stable operation mode of the mineral water field's setting problem is the development and implementation of the hydromineral recourses distributed control system. In this article the consideration of the control system's development on the basis of regulator synthesis approach is offered. The purpose of this study is to present a technique of the distributed regulator synthesis for the mineral water production process' control system. At the beginning of the work, approaches of the management problem solution for concentrated and distributed control systems are considered. The problem definition for object with the distributed parameters, described by the system of the linear differential equations is executed, the description of management object's mathematical model is carried out, entry and boundary conditions are defined. The technique of transfer function definition is shown on a practical example, the model of management is considered by process of mineral water production. It is shown that entrance influence can be presented in the form of Fourier's number, for invariant systems function of an exit will correspond to entrance influence. Transfer function is defined in the form of the set of transfer functions, corresponding to each harmonica of entrance influence. The offered mechanism allows to offer a method of transfer function calculation for each harmonica of entrance influence separately that significantly simplifies process of the analysis of the control system. The main result is the possibility of representation of the system in the form of the set of transfer functions.
\end{abstract}

Keywords: Control Systems, Mathematical Modeling, Geofiltration Models, Aquifer Pressure Function, Synthesis of the Distributed Parameters Controller

\section{INTRODUCTION}

In the modern world the problem of rational subsurface using is particularly acute. From the medical point of view one of the most valuable resources are mineral waters, therefore providing the stable conditions of their production is an important task (Chernyshev et al., 2014). One of the ensuring mineral water field's stable operation ways is the development of the control system on the basis of controller synthesis (Martirosyan et al., 2013). Also, the different methods of the distributed system's synthesis are exist
(Finkbeiner and Peter, 2012), but if we regard mineral water fields as the object of control, the controller's synthesis is considered. When using this way, mineral water field's can be regarded like concentrated or distributed systems.

The concentrated systems are the systems which movement can be described as a movement of final number of objects with rigidly fixed internal structure. The method of concentrated systems means the representation of management object as a set of elements, that are not dependent from each other (Rapoport, 2005). For example, in relation to mineral Corresponding Author: Alexander V. Martirosyan, North-Caucasian Federal University, branch in Pyatigorsk, Russian Federation, 357500, Pyatigorsk, 40 let Oktyabrya ave., 56A, Russia 
water fields it is the in taking and control wells. The system is considered as concentrated in the case when it is possible to neglect the segment's influence to each other in the system. When communications between elements are rather essential, the methods of the distributed parameters systems' synthesis are applied. The main differences of these type systems from the concentrated systems are: The decentralized management and the presence of spatial coordinates at object's mathematical model.

The main forms of the distributed objects (systems) representation, as well as in case of systems with the concentrated parameters, are the representation in the form of the differential equations in private derivatives, representation in the form of transfer functions, representation in the form of temporary characteristics and representation in the form of frequency characteristics (Miller and Michel, 2007).

Let's set the task of object's reaction definition on the own vector functions of the object's operator. In this case the distributed object (system) can be structurally presented as the infinite set of the independent conditionally concentrated contours. Transfer function of the each of their conditionally concentrated contour can be presented in the form of the analytical entire functions' relation (Kalyon, 2010).

In the case when own vector functions are presented on the basis of orthogonal decomposition to Fourier's series on spatial coordinates, the class of spatial and invariant objects and systems, for which the technique of the distributed controller synthesis is developed can be allocated. The technique of the distributed controller synthesis allows to receive the controller for the considered control system and to estimate its stability (Kravcova et al., 2012).

\section{MATERIALS AND METHODS}

Let's carry out the first stage of distributed controller's synthesis, assuming definition of transfer function of system. Let's consider object with the distributed parameters, described by the system of linear differential equations in private derivatives:

$$
\begin{aligned}
& \frac{\partial T_{i}}{\partial \tau}=L_{i}\left(T_{i} ; \frac{\partial T_{i}}{\partial x} ; \ldots \frac{\partial^{n_{1}} T_{i}}{\partial x^{n_{1}}} ; \frac{\partial T_{i}}{\partial y} ; \ldots \frac{\partial^{n_{2}} T_{i}}{\partial y^{n_{2}}} ; \frac{\partial T_{i}}{\partial z} ; \ldots \frac{\partial^{n_{3}} T_{i}}{\partial z^{n_{3}}}\right), \\
& x, y, z \in V, \quad(i=\overline{1, n})
\end{aligned}
$$

Where:

$T_{i}(x, y, z, \tau)=$ Phase variable $(i=\overline{1, n})$

$$
\begin{array}{ll}
x, y, z & =\text { Corresponded coordinates } \\
\tau & =\text { Time } \\
V & =\text { Space of variable's changing } x, y, z \\
N, n_{1}, n_{2}, n_{3} & =\text { Predetermined integer value } \\
L_{i} & =\text { Linear operators }
\end{array}
$$

The object of automatic control presented in the form of 1.1 , is called spatial and invariant if the complex transfer coefficient on each component of an input action's number doesn't depend on spatial coordinates. At physical level this definition means that each component of an input action's number, passing through management object, changes only amplitude of spatial fashion. Boundary conditions for the equation system (1.1) are taking to be homogeneous and given as:

$$
\begin{aligned}
& L_{z, i}\left[T_{i}(x, y, z, \tau), T_{i+1}(x, y, z, \tau)\right]=0 ; \\
& x, y, z \in \Gamma_{1, i}, \quad(i=1, n-1) ; \\
& \tilde{L}_{z, \tilde{j}}\left[T_{i}(x, y, z, \tau)\right]=0 ; \\
& x, y, z \in \Gamma_{2, \tilde{j}}, \quad(i=\overline{1, n} ; \quad j=1, \tilde{n}) ; \\
& \tilde{\tilde{L}}_{z, \mu}\left[\begin{array}{c}
* \\
T_{\mu} \\
\left.\left.x, y, z_{\mu}, \tau\right), \quad U_{\mu}(x, y, \tau)\right]=0 ;
\end{array}\right] \\
& x, y \in \Gamma_{3, \mu}, \quad(\mu=\overline{1, m}),
\end{aligned}
$$

Where:

$$
\begin{aligned}
& L_{2, i}, \tilde{L}_{2, j}, \tilde{\tilde{L}}_{2, \mu} \\
& (i=\overline{1, n-1} ; j=\overline{1, \tilde{n} ; \mu}=\overline{1, m}) \quad=\text { Linear operators } \\
& \Gamma_{1, i}, \Gamma_{2, \tilde{j}}, \Gamma_{3, \mu}, \\
& (i=\overline{1, n-1} ; \tilde{j}=\overline{1, \tilde{n}} ; \mu=\overline{1, m}) \quad=\text { Boundary subareas of } \\
& \tilde{n}, m \\
& { }^{*}, \quad(\mu=\overline{1, m}) \\
& \text { the } V \text { space } \\
& =\text { Predetermined values } \\
& =\text { Fixed value of } z \\
& \text { coordinate } \\
& \mathrm{U}_{\mu}(\mathrm{x}, \mathrm{y}, \tau),(\mu=\overline{1, \mathrm{~m}}) \\
& \text { Input action }
\end{aligned}
$$

Object's output functions are the values of phase variables $T_{i}\left(x, y, \bar{z}_{i}, \tau\right)$ with fixed values of $z=\bar{z}_{i}(\mathrm{i}=\overline{1, \mathrm{n}})$. 
Further it is offered to consider the distributed objects which mathematical models which allow the expansion on the own vector functions of the object's operator. Using such expansion, transfer function of the distributed object can be provided by a set of transfer functions on spatial modes.

\section{RESULTS}

As an example of practical using, the follows field's geofiltration model with $i$-number of layer with the $\eta^{*}$ compressibility coefficient is presented. The graphical scheme of the model is as follows (Fig. 1).

Mathematical model of the layers' water expansion (Fig. 1) can be presented in a look:

$$
\begin{aligned}
& \eta \cdot \frac{\partial H_{i}}{\partial \tau}=\frac{\partial^{2} H_{i}}{\partial x^{2}}+\frac{\partial^{2} H_{i}}{\partial y^{2}}+\frac{\partial^{2} H_{i}}{\partial z^{2}}, \quad(i=\overline{1, n}) \\
& z_{i}<z<z_{i-1}, \quad 0<x<x_{L}, \quad 0<y<y_{L},
\end{aligned}
$$

Where:

$$
\begin{array}{ll}
H_{i}(x, y, x, \tau) & \text { Pressure in the } i \text { layer of the } \\
& \text { field } \\
z_{i}(i=\overline{0, n+1}), n, x_{L}, y_{L}, a_{i}= & \text { Predetermined values } \\
(x, y, z) & \text { Corresponded coordinates } \\
\tau & =\text { Time } \\
\eta^{*} & =\text { Predetermined coefficient }
\end{array}
$$

On the boundaries of the layers contiguity the condition of pressure $(H)$ and derivative of $H$ for the corresponding coordinate $z$ :

$$
\begin{aligned}
& H_{i}\left(x, y, z_{i}, \tau\right)=H_{i+1}\left(x, y, z_{i}, \tau\right), \quad(i=\overline{1, n-1}) \\
& \lambda_{i} \frac{\partial H_{i}\left(x, y, z_{i}, \tau\right)}{\partial z}=\lambda_{i+1} \frac{\partial H_{i+1}\left(x, y, z_{i}, \tau\right)}{\partial z}, \quad(i=\overline{1, n-1}) .
\end{aligned}
$$

where, $\lambda_{i}$ Predetermined values

On a lateral surface of a conventional field (Fig. 1) these condition are taking place:

$$
\begin{aligned}
& T_{i}(x, y, z)=0, \quad(i=\overline{1, n}) \\
& x, y, z, \in S_{\mu}, \quad(\mu=\overline{1,6}),
\end{aligned}
$$

where, $S_{\mu}$ Lateral surfaces of a field $(\mu=\overline{1,6})$ (Fig. 1).
Input action $U(x, y, \tau)$ is distributed in the $i^{*}$-layer $\left(i^{*}-\right.$ fixed integer $\left.\left(i{ }^{*} \leq n\right)\right)$ and can be presented like:

$H_{i^{*}}(x, y, \stackrel{*}{*}, \tau)=U(x, y, \tau)$

where, ${ }^{*}$ fixed value $\mathrm{z},{ }^{*} \in\left[z_{i^{*}}, z_{i^{*}-1}\right]$

Function of an output is the value of a function $H_{\bar{n}}(x, y, \bar{z}, \tau)$, where $\bar{n}, \bar{z}-\bar{n}, \bar{z}$ - - the fixed value of $i$ $(\bar{n} \in[\overline{1, n}])$ parameter and $\mathrm{z}$ coordinate respectively.

After input influence and an output function are been defined, it is offered to define transfer function for the distributed control system. Let's consider a mathematical model of a field which is shown in a Fig. 1. This model is described by the Equation 1.1-1.6.

Initial conditions for the equation 1.7 are presented like:

$$
H(x, y, z, 0)=0 .
$$

Boundary conditions for the Equation 1.7 are presented like Equation 1.8:

$$
\begin{aligned}
& H(0, y, z, \tau)=H(x, 0, z, \tau)=H\left(x_{L}, y, z, \tau\right) \\
& =H(x, y, z, \tau)=0 ; \\
& \frac{\partial H(x, y, 0, \tau)}{\partial z}=0 ; \\
& H\left(x, y, z_{L}, \tau\right)=U(x, y, \tau) .
\end{aligned}
$$

Input function is the value of the pressure function $H\left(x, y, z={ }^{*}, \tau\right)$.

where, ${ }^{*}$ Predetermined value $\left(0<z z_{L}\right)$

Let's spread out the input action $U(x, y, \tau)$ to a Fourier's series. Considering boundary conditions (1.8), the input action can be presented in a look:

$$
U(x, y, \tau)=\sum_{\eta, \gamma=1}^{\infty} C_{\eta, \gamma}(\tau) \cdot \sin \left(\psi_{\eta} \cdot x\right) \cdot \sin \left(\tilde{\phi}_{\gamma} \cdot y\right)
$$

Where:

$$
\begin{aligned}
& \psi_{\eta}=\pi \cdot \eta / x_{L} ; \tilde{\phi}_{\gamma}=\pi \cdot \gamma / y_{L} \\
& \psi_{\eta}, \tilde{\phi}_{\gamma}=\text { Spatial frequency }
\end{aligned}
$$




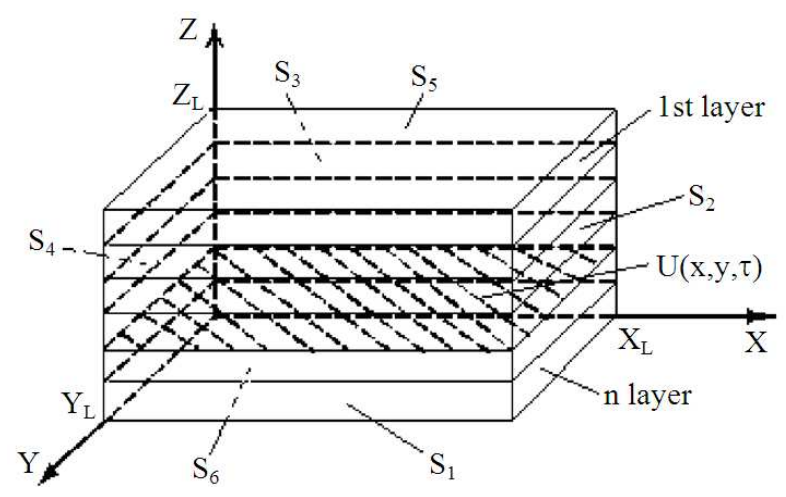

Fig. 1. The scheme of the modeled field

Let's the find reaction of the each object's component of a row (1.7). This reaction is looked for in a look:

$$
T_{\eta, \gamma}(x, y, z, \tau)=B_{\eta, \gamma}(z, \tau) \cdot \sin \left(\psi_{\eta} \cdot x\right) \cdot \sin \left(\tilde{\phi}_{\gamma} \cdot y\right)
$$

Substituting (1.8) to the Equation (1.2) and transforming them the following result will be Equation 1.11:

$$
\begin{aligned}
& \frac{\dot{B}_{\eta, \gamma}(z, \tau)}{a}+B_{\eta, \gamma}(z, \tau) \cdot\left(\psi_{\eta}^{2}+\tilde{\phi}_{\gamma}^{2}\right) \\
& -\frac{\partial^{2} B_{\eta, \gamma}(z, \tau)}{\partial z^{2}}=0,(\eta, \gamma=\overline{1, \infty})
\end{aligned}
$$

Then the transformation (4.9) according to Laplace under the zero initial conditions is offered Equation 1.12:

$$
\begin{aligned}
& -\frac{\partial^{2} \bar{B}_{\eta, \gamma}(z, s)}{\partial z^{2}}+\left(\frac{s}{a}+\psi_{\eta}^{2}+\tilde{\phi}_{\gamma}^{2}\right) \cdot \bar{B}_{\eta, \gamma}(z, s) \\
& =0,(\eta, \gamma=\overline{1, \infty})
\end{aligned}
$$

where, $\quad \bar{B}_{\eta, \gamma}(z, s)$ the function's $B_{\eta, \gamma}(z, \tau)$ Laplace transform under the zero initial conditions $(\eta, \gamma=\overline{1, \infty})$.

The (1.10) equation's solution can be submitted in a look Equation 1.13:

$$
\begin{aligned}
& \bar{B}_{\eta, \gamma}(z, s)=\Omega_{1, \eta, \gamma} \cdot \exp \left(\beta_{\eta, \gamma} \cdot z\right) \\
& +\Omega_{2, \eta, \gamma} \cdot \exp \left(-\beta_{\eta, \gamma} \cdot z\right),(\eta, \gamma=\overline{1, \infty})
\end{aligned}
$$

where, $\beta_{\eta, \gamma}=\left(\frac{s}{a}+\psi_{\eta}^{2}+\tilde{\phi}_{\gamma}^{2}\right)^{1 / 2},(\eta, \gamma=\overline{1, \infty})$.
From the (1.4), (1.5) boundary conditions the follows will be in a look Equation 1.14:

$$
\begin{aligned}
& \Omega_{1, \eta, \gamma}=\Omega_{2, \eta, \gamma} ; \\
& \Omega_{2, \eta, \gamma}=\frac{\bar{C}_{\eta, \gamma}(s)}{\exp \left(\beta_{\eta, \gamma} \cdot z_{L}\right)+\exp \left(-\beta_{\eta, \gamma} \cdot z_{L}\right)},(\eta, \gamma=\overline{1, \infty})
\end{aligned}
$$

where, $\bar{C}_{\eta, \gamma}(s)$-the function's $C_{\eta, \gamma}(\tau)$ Laplace transform under the zero initial conditions $(\eta, \gamma=\overline{1, \infty})$.

Considering (1.12), (1.13), (1.14) in common and taking into account the (1.9), the following results can be received Equation 1.5:

$$
\begin{aligned}
& \bar{H}_{\eta, \gamma}(x, y, z, s) \\
& =\frac{\exp \left(\beta_{\eta, \gamma} \cdot z\right)+\exp \left(-\beta_{\eta, \gamma} \cdot z\right)}{\exp \left(\beta_{\eta, \gamma} \cdot z_{L}\right)+\exp \left(-\beta_{\eta, \gamma} \cdot z_{L}\right)} \\
& \bar{C}_{\eta, \gamma}(s) \cdot \sin \left(\psi_{\eta} \cdot x\right) \cdot \sin \left(\tilde{\phi}_{\gamma} \cdot y\right)
\end{aligned}
$$

where, $\bar{H}_{\eta, \gamma}(x, y, z, s)$-Laplace transformation of the $H_{\eta, \gamma}(x, y, z, \tau)$ function.

Object's transfer function in $\eta, \gamma \quad(\eta, \gamma=\overline{1, \infty})$ input action's mode can be submitted in a look Equation 1.6:

$$
\begin{aligned}
& W_{0, \eta, \gamma}(s)=\frac{\bar{H}_{\eta, \gamma}\left(x, y, z={ }^{*}, s\right)}{\bar{C}_{\eta, \gamma}(s) \cdot \sin \left(\psi_{\eta} \cdot x\right) \cdot \sin \left(\tilde{\phi}_{\gamma} \cdot y\right)}= \\
& \frac{\exp \left(\beta_{\eta, \gamma} \cdot z\right)+\exp \left(-\beta_{\eta, \gamma} \cdot z\right)}{\exp \left(\beta_{\eta, \gamma} \cdot z_{L}\right)+\exp \left(-\beta_{\eta, \gamma} \cdot z_{L}\right)} \\
& (\eta, \gamma=\overline{1, \infty})
\end{aligned}
$$

Thus, the considered distributed object can be presented in the form of the set of transfer functions $W_{0, \eta, \gamma}(s) \quad(\eta, \gamma=\overline{1, \infty})$.

\section{DISCUSSION}

Transfer function can be calculated for each Fourier component which represents the input influence separately. It allows the implementation of the mineral water production control system's 
synthesis process (Martirosyan and Martirosyan, 2014). The technique of the regulator synthesis consists of determination of transfer function's numerical parameters for open-ended system by the means of graphic-analytical methods (Schewe, 2008). Further, the system is shorted and the found parameters are used for adjustment of input influence. The parameters of closed system calculated with this way, will show the stability of regulation process. The volume of this article doesn't allow to show all process of the distributed system's controller synthesis. In this article the only the algorithm of transfer function finding was shown. That is the intermediate step in distributed controller's synthesis for the control system.

The technique of the controller for the distributed control system's synthesis was applied to mineral water fields and gives adequate results (Malkov and Pershin, 2007), but, despite it, there are a certain problems of practical application of the specified technique. The main problem is the difficulties of the technique application in case of the controlled object's (field) difficult structure or the existence of a large number of external factors which are influencing on the research processes inside a field. The problem is that the accounting of each similar process is impossible, because of the large volume of processed data and using of average coefficients leads to the computation error increasing. The main objective of future researches can be formulated as complication of a models structure for the purpose of more exact compliance to a difficult gidrogelogical section of a specific field.

\section{CONCLUSION}

In article the technique of the controller for the distributed control system's synthesis was considered by process of mineral water production. Input influence was set and the output function that gave the chance to define a transfer function of control system is defined. Expansion of the input influence in a Fourier series was made and the type of the object's response on each component of a row is defined. Further, by substitution of the object's response equation in a mathematical model and conversions of the received result. The solution of the equation was received. Determination of a type of the transfer function on a certain mode of the input influence became the total of conversions. Received results shows, that the distributed object can be considered in the form of the set of transport functions. This method of a control object representation allows to approach to the analysis of control systems from other side and to improve process of their analysis. The technique explained in article, is one of the stages of the controller for the distributed control system's synthesis, can be applied to control the rate of flow of the mineral water fields and proved to be in practice.

\section{ACKNOWLEDGMENT}

The researchers acknowledge receiving support from the North-Caucasus Federal University represented by Dr. Ivan Pershin and Dr. Tatyana Shebzukhova. We are responsible for all errors as well as heavy style of the manuscript.

\section{REFERENCES}

Chernyshev, A.B., K.V., Martirosyan and A.V., Martirosyan, 2014. Analysis of the nonlinear distributed control system's sustainability. J. Math. Stat., 10: 316-321. DOI: 10.3844/jmssp.2014.316.321

Finkbeiner, B. and H.J. Peter, 2012. Template-based controller synthesis for timed systems. Proceedings of the 18th International Conference on Tools and Algorithms for the Construction and Analysis of Systems, Mar. 24-Apr. 1, Springer Berlin Heidelberg, Tallinn, Estonia, pp: 392-406. DOI: 10.1007/978-3-642-28756-5_27

Kalyon, G., T. Le Gall, H. Marchand and T. Massart, 2010. Synthesis of communicating controllers for distributed systems. Proceedings of 50th IEEE Conference on Decision and Control and European Control Conference, Dec. 12-15, IEEE Xplore Press, Orlando, FL., pp: 13-17. DOI: 10.1109/CDC.2011.6160584

Kravcova, A.L., J.V. Ilushin and M.M. Mardoyan, 2012. Stability of the distributed control system temperature pattern. Science Education, Moscow.

Malkov, A.V. and I.M. Pershin, 2007. Distributed controller synthesis for the hydrolitosphere processes management systems. Science World, Moscow.

Martirosyan, K.V., A.V. Martirosyan and T.S. Kapylova, 2013. The model of mineral water deposits sustainable management using the decision support system. World Applied Sci. J. 
Martirosyan, A.V. and K.V. Martirosyan, 2014. Ensuring sustainable model of mineral water deposits operating modes on the example of the deposit Nagutskaya. Int. Sci. Technical J., 6: 89-93.

Miller, R.K. and A.N. Michel, 2007. Ordinary Differential Equations. 1st Edn., Dover Publications, USA, pp: 250.
Rapoport, E.J., 2005. Analysis and Synthesis of the Automatic Control Systems with Distributed Parameters. 1st Edn., Higher School, Moscow, pp: 292.

Schewe, S., 2008. Synthesis of distributed systems. PhD Thesis, Date Views. 